\title{
Open Science, aber richtig! \\ Was wir aus der Heinsberg-Studie lernen können
}

\section{Ein Kommentar}

Autor*innen (laut $\underline{\mathrm{CRediT}}$, alphabetisch)*

Konzept und Initiierung:

Caroline Fischer, ORCID iD: 0000-0001-5870-4498, Universität Potsdam

Jo Havemann, ORCID iD: 0000-0002-6157-1494, Access 2 Perspectives

Tamara Heck, ORCID iD: 0000-0001-5519-6395, Leibniz-Institut für Bildungsforschung und

Bildungsinformation

Erstentwurf:

Hans Henning Stutz, ORCID iD: 0000-0003-3360-1243, Aarhus University, Department of Engineering

Nate Breznau, 0000-0003-4983-3137, Universität Bremen

Tamara Heck, ORCID iD: 0000-0001-5519-6395, Leibniz-Institut für Bildungsforschung und

Bildungsinformation

Katja Mayer, ORCID iD: 0000-0003-1184-595X, Universität Wien

Isabella Peters, ORCID iD: 0000-0001-5840-0806, Leibniz-Informationszentrum Wirtschaft \& Christian-

Albrechts-Universität zu Kiel

Philipp Schrögel, ORCID ID: 0000-0003-0892-8703, Karlsruher Institut für Technologie

Revisionen:

Nate Breznau, ORCID iD: 0000-0003-4983-3137, Universität Bremen

Caroline Fischer, ORCID iD: 0000-0001-5870-4498, Universität Potsdam

Jo Havemann, ORCID iD: 0000-0002-6157-1494, Access 2 Perspectives

Tamara Heck, ORCID iD: 0000-0001-5519-6395, Leibniz-Institut für Bildungsforschung und

Bildungsinformation

Katja Mayer, ORCID iD: 0000-0003-1184-595X, Universität Wien

Isabella Peters, ORCID iD: 0000-0001-5840-0806, Leibniz-Informationszentrum Wirtschaft \& Christian-

Albrechts-Universität zu Kiel

Philipp Schrögel, ORCID ID: 0000-0003-0892-8703, Karlsruher Institut für Technologie

Hans Henning Stutz, ORCID iD: 0000-0003-3360-1243, Aarhus University, Department of Engineering

* Zu dieser Veröffentlichung gibt es die englische Version Open Science, but Correctly! Lessons from the Heinsberg Study auf MetaArXiv. Die Autor*innen sind Open Science-Advokat*innen des Wikimedia Fellow-Programms Freies Wissen (2019/2020). Die Idee zum Beitrag wurde in dieser Forschenden-Gemeinschaft initiiert. Die Autor*innen bedanken sich bei den anderen Open Science-Advokat*innen für zahlreiche Kommentare und Feedback zum Beitrag.

In Zeiten von Corona wird der Ruf nach Zugang zu akademischem Wissen, öffentlich zugänglichen Daten und Studien und einer transparenten Kommunikation immer lauter - kurzum, 'Open Science' ist Teil der öffentlichen Debatte geworden. Gleichzeitig werden genau diese Open Science-Praktiken in der Öffentlichkeit vielfach kritisiert bzw. aus dem Kontext gerissen dargestellt. Wir wollen daher anhand der laufenden Debatten im Spannungsfeld zwischen einer Öffnung der Wissenschaft und einem autoritäten- und faktenbasierten Krisenmanagement den Herausforderungen von guter Open Science-Praxis nachgehen, und die Kritik aber auch die positiven Möglichkeiten für ein neues Wissenschaftsverständnis in den Fokus rücken. 
Die Krise hat dazu geführt, dass mehr öffentliche Aufmerksamkeit auf der zeitnahen Kommunikation relevanter Forschungsergebnisse liegt. Menschen suchen nach Wissen, aber auch nach Sicherheiten und Entscheidungsgrundlagen. In Deutschland wurden wissenschaftliche Prozesse und Ergebnisse in den Medien so präsent wie selten zuvor diskutiert, beispielsweise die sogenannte "Heinsberg-Studie" und die Studie zur Viruslast bei Kindern vom CharitéVirologen Christian Drosten. Sie wurden dort aber nicht nur als lang ersehnte Erkenntnisse willkommen geheißen, sondern von verschiedenen Seiten stark kritisiert, unter anderem aufgrund ihrer wissenschaftlichen Qualität und Aussagekraft, aber auch wegen der Art der Präsentation und Kommunikation der vorläufigen Ergebnisse (siehe beispielhaft den Bericht des BR, zum Kontext ein Interview mit Drosten in der Süddeutschen).

In Krisensituationen wird das generelle Spannungsverhältnis zwischen Verbreitungsgeschwindigkeit und Qualität wissenschaftlicher Erkenntnisse besonders sichtbar. Dabei sind zentrale Prinzipien von Open Science gerade die größtmögliche Transparenz und Öffnung des Wissens für einen breiten Diskurs.

Was können wir nun aus den Debatten über wissenschaftliche Integrität in Krisenzeiten für eine gute Open Science-Praxis lernen? Wie können wir möglichst offen agieren, dabei aber umsichtig mit den Erwartungshaltungen in der Öffentlichkeit umgehen?

Zentral sind dabei die folgenden Aspekte:

- Geschwindigkeit der Veröffentlichung,

- Qualitätssicherung und Verfügbarkeit von kritischen wissenschaftlichen Informationen,

- medialisierte Kommunikation.

\section{Ein exemplarischer Rückblick: Die "Heinsberg-Studie"}

Anfang April diesen Jahres hat der Virologe Hendrik Streeck mit Kolleg*innen die Zwischenergebnisse der "Heinsberg-Studie" auf einer Pressekonferenz präsentiert. Diese Präsentation erfolgte bereits vor Veröffentlichung eines Manuskripts und der Forschungsdaten. Erst Anfang Mai wurde ein erster Preprint-Artikel dazu auf medRxiv hochgeladen, der Anfang Juni aktualisiert wurde. Darin ist auch vermerkt, dass die Forschungsdaten auf Anfrage zu erhalten seien. Letzteres ist üblich, da sensible Daten wie Gesundheitsdaten Forschenden nur auf Anfrage zugänglich sind. Aus datenschutzrechtlichen Gründen werden sie jedoch nicht gänzlich offen im Netz publiziert. Aktuell (02.07.2020) liegt noch kein Peer-Review der Studie vor. Andere Wissenschaftler*innen kritisierten in der öffentlichen Diskussion, dass die Forschenden "nur" Zwischenergebnisse hatten, kein wissenschaftliches und geprüftes Manuskript und keine Forschungsdaten vorlagen, und die knappen Zwischenergebnisse erstmals auf einer nationalen Pressekonferenz präsentiert wurden.

\section{Die Ziele von Open Science}

Ist das Vorgehen in diesem Fall im Sinn von Open Science, und steht es im Widerspruch zu den klassischen wissenschaftlichen Werten? Keineswegs: Open Science verlangt die konsequente 
Umsetzung guter wissenschaftlicher Praxis. Eine Offene Wissenschaft hat aber auch das Ziel, Wissenschaft noch stärker als bisher als Gemeinschaft zu betreiben. Daher ist die Leitidee der Open Science-Bewegung, wissenschaftliche Prozesse - von den Ideen, über die Methoden, Analysen und Daten, hin zu den Ergebnissen - so früh wie möglich zu veröffentlichen. Das Prinzip lautet dabei aber bewusst nicht 'publishing as fast as possible', sondern 'sharing knowledge as early as possible'.

Dahinter steckt die Idee, dass die frühestmögliche Veröffentlichung wissenschaftlicher Prozesse eine breite Öffentlichkeit generiert. Dies fördert die Diskussion, welche wiederum zu einer Verbesserung der Forschung führt. Dabei gilt es zu bedenken, dass wissenschaftliche Erkenntnisse immer mit einer gewissen Unsicherheit und Vorläufigkeit verbunden sind und ausdrücklich der Diskurs mit Fachkollegen und Akteuren der Gesellschaft gesucht wird.

Gerade wenn das Wissen bereits im Entstehungsprozess geprüft und besprochen wird, werden vorhandene Ressourcen am effektivsten eingesetzt. In Krisenzeiten kann man nicht - wie üblich - monatelang bis zu zwei Jahre auf die Veröffentlichung in einer akademischen Zeitschrift warten, um Forschungsergebnisse eingehend zu prüfen und zu kritisieren.

Viele COVID-19 Forschende folgen diesem Open Science-Prinzip und veröffentlichen ihre Daten und Ergebnisse so schnell wie möglich; Forschungsdaten und wissenschaftliche Manuskripte werden auf gemeinschaftlichen Online-Plattformen für COVID-19 Daten geteilt und gesammelt.

Auch die Forschenden der "Heinsberg-Studie" wollten schnell (Zwischenergebnisse) und breit (groß angelegte Pressekonferenz) über ihre Erkenntnisse informieren. Dennoch hagelte es Kritik an diesem Vorgehen, in einzelnen Aspekten der Vorgehensweise unserer Einschätzung nach durchaus berechtigt.

\section{Geschwindigkeit vs. Qualität}

Nach einer Schätzung umfasst die seit Januar 2020 zu COVID-19 veröffentlichte Literatur mehr als 25.000 Manuskripte, wobei sich die Menge alle 20 Tage verdoppelt. Damit wird die Öffentlichkeit Zeugin einer der größten Explosionen von wissenschaftlicher Literatur in der neueren Zeit.

Sogenannte Preprint-Server oder Repositorien wie preprints.org, OSF-preprints, bioRxiv oder medRxiv - ermöglichen die Publikation in einer Geschwindigkeit, die in der print-getriebenen Veröffentlichungspraxis bisher nicht üblich war. Auch viele Forschungsförderer unterstützen derzeit beschleunigte Veröffentlichungsprozesse. Die schnelle Verbreitung von Ergebnissen oder auch Zwischenergebnissen ist in der offenen Wissenschaft erwünscht, wenn diese gut fundiert sind, und alle Voraussetzungen erfüllen um ein Begutachtungsverfahren (Peer-Review) durch Fachkollegen zu ermöglichen. So können solche ohne ausführlichen Begutachtungsprozess validierten Erkenntnisse nämlich als Informationsgrundlage für andere Forschende dienen, weitere Forschung ankurbeln, Redundanzen vermeiden und für die Politik unter Vorbehalt richtungsweisende Informationsgrundlagen bereitstellen. 
Doch zu viel Unsicherheit, oder gar falsche Ergebnisse, können auch großen Schaden anrichten, gerade wenn daraus durch die Politik gesellschaftsrelevante Entscheidungen abgeleitet werden. Die Akteure haben rasch gelernt: Einige Plattformen wie bioRxiv und medRxiv änderten ihre üblichen Qualitätssicherungsprozesse und begannen stärker zu reglementieren; beispielsweise werden eingereichte Preprints stärker und gezielt geprüft, besonders wenn es sich um medizinische Forschung handelt, die Auswirkungen auf Therapien haben könnte, oder komplexe statistische Analysen beinhalten.

In traditionellen Peer-Review-Verfahren prüfen Fachexpert*innen die Forschungsprozesse (u.a. Methoden, erhobene Daten) und -ergebnisse ihrer Kollegen*innen bevor die Ergebnisse veröffentlicht werden. In den Natur- und Lebenswissenschaften werden Zwischenergebnisse zusätzlich auf wissenschaftlichen Konferenzen besprochen, wo sie ein erstes formales PeerReview durchlaufen. Nach einem weiteren Peer-Review erfolgt die Veröffentlichung beispielsweise in einer wissenschaftlichen Zeitschrift.

Die Wissenschaft hat mit dem Peer-Review ein wichtiges Instrument zur Prüfung von Forschungsmethoden, -analysen, und -ergebnissen etabliert - wenn auch das traditionelle PeerReview-Verfahren seine Schwächen hat (siehe hierzu: Rödel, 2020; Interview mit Ross-Hellauer).

Auf Preprint-Servern sind die meisten Publikationen zuvor noch keinem Peer-Review unterzogen worden, damit sie schneller anderen Forschenden zugänglich gemacht werden können und sich das Wissen verbreitet. Sie werden also erst nachträglich in einem Post-Publication Peer-Review öffentlich diskutiert und begutachtet. Betrachtet man Preprints als Werkzeug, das die Befolgung des Prinzips der frühestmöglichen Veröffentlichung sicherstellt, und klassisches Pre-Publication Peer-Review als Werkzeug, um das Prinzip der Qualitätssicherung zu gewährleisten, wird klar, dass das eine Prinzip mitunter zu Lasten des anderen geht.

Dennoch ist zu betonen, dass Preprint-Manuskripte von der Wissenschaft direkt gelesen und nachvollzogen und mit Zugabe von Forschungsdaten auch transparent geprüft werden können. Dies scheint bei der "Heinsberg-Studie" nicht geschehen. Die verteidigende Aussage von Hendrik Streeck, dass eine Preprint-Veröffentlichung 2 bis 3 Wochen gedauert hätte, ist jedoch nicht ganz von der Hand zu weisen, da Preprint-Server in der aktuellen Situation zum Teil mit Anfragen überlastet sind. Und Forschungsdaten sollten auch entsprechend nachvollziehbar für andere Forschende aufbereitet werden, was wiederum Zeit kostet. Im Sinne von Open Science sind beide Aspekte aber wichtig, um Transparenz, Nachvollziehbarkeit und Prüfbarkeit von Forschung zu gewährleisten - Punkte, die auch beim Prinzip der frühestmöglichen Veröffentlichung nicht vernachlässigt werden dürfen.

Nun finden in Corona-Zeiten u. U. Konferenzen nicht oder nicht mehr in ausreichendem Umfang statt und sind auch sonst nicht immer dann verfügbar, wenn ein schnelles Feedback von Kolleg*innen erforderlich ist. Was könnte man also in Zukunft tun, um Forschungsergebnisse schnell zu veröffentlichen und sie dennoch auf ihre Qualität hin zu prüfen? 
Denkbar wäre ein ergänzender Qualitätssicherungsmechanismus, wie ein Expert*innenbeirat, der sich ad-hoc bildet und Feedback gibt. Hierfür könnten Fachgruppen oder übergreifende Organisationen, wie die Deutsche Forschungsgemeinschaft einen Pool aus Wissenschaftler*innen bilden, die, ähnlich wie beim formalen Peer-Review, für kurzfristige Begutachtungen angefragt werden können. Wer Drittmittelprojekte beantragt und durchführt, weiß, dass ein solches Gremium häufig einberufen wird. Nicht umsonst. Es sollte auf solche Lösungen auch für die Auftragsforschung gesetzt werden, wenn sie von solcher Bedeutung ist, wie es die "Heinsberg-Studie" war.

Im Sinne eines Open-Peer-Review muss hier diskutiert werden, inwiefern diese ad-hoc Begutachtungen auch veröffentlicht werden, um die Qualitätssicherungsmaßnahmen transparent zu machen.

\section{Verfügbarkeit kritischer wissenschaftlicher Informationen: Offene Daten als "Muss"}

Gemäß dem Prinzip FAIR - findable, accessible, interoperable, reusable - strebt eine offene Wissenschaft stets nach der größtmöglichen Transparenz und Prüfbarkeit des Forschungsprozesses. Denn Open Science will nicht nur Ergebnisse zugänglich machen: Erst das Teilen von vorläufigen und endgültigen Forschungsdaten, aber auch von Methoden, Protokollen und Standards macht eine Überprüfung der Ergebnisse überhaupt möglich. Dies ist besonders wichtig, wenn die Ergebnisse noch nicht durch einen Peer-Review-Prozess validiert wurden, ist aber ohnedies immer wünschenswert.

Im Fall der "Heinsberg-Studie" waren die Daten zum Zeitpunkt der Pressekonferenz nicht verfügbar, eine rasche, Beurteilung ihrer Qualität damit nicht möglich. Das entspricht nicht der Idee von Open Science und ist zu kritisieren. Dass eine direkte vollständige Transparenz höhere zeitliche Kapazitäten der Forschenden verlangt und es besserer technischer Lösungen bedarf, ist eine Herausforderung, die es in Zukunft anzugehen gilt.

Die Kritik am Fehlen offener oder teil-offener Daten richtet sich derzeit aber nicht nur an die Wissenschaft. Auch die öffentliche Hand ist gefordert, möglichst rasch und unter Wahrung des Datenschutzes, relevante Informationen zu sammeln und zur Verfügung zu stellen. Die Krise zeigt, wie wenig die dafür notwendigen Protokolle und Infrastrukturen noch entwickelt sind und dass ein großer Handlungsbedarf besteht. Die Wissenschaft kritisiert hierbei zu Recht die schlechte Qualität und fehlende Nachvollziehbarkeit von zugänglichen Gesundheitsdaten und deren Kontextinformationen - diese Daten sind nämlich häufig nicht FAIR. Die für Fragen der öffentlichen Gesundheit so wichtige Verknüpfbarkeit und Interoperabilität können so nicht gewährleistet werden.

\section{Medialisierte Kommunikation: Offenheit deckt Interessen von Akteuren auf}

Open Science betrifft auch die Art und Weise, wie wissenschaftliche Erkenntnisse an und in diversen Öffentlichkeiten kommuniziert werden. Dies betrifft neben traditionellen 
wissenschaftlichen Publikationsformaten wie Wissenschaftsmagazine auch die Massenmedien und digitalen Öffentlichkeiten.

Offene Kommunikation und gleichzeitige Qualitätsprüfung funktioniert innerhalb der Wissenschaftsnetzwerke schon sehr gut. Ein gutes Beispiel ist die eingangs erwähnte Studie der Charité. Sie wurde als Pre-Print Version veröffentlicht, woraufhin andere Forschenden die Verantwortlichen auf Fehler aufmerksam gemacht haben. Beide Seiten tauschten sich aus und die Ergebnisse der Studie wurde überarbeitet. Dieser Vorgang ist in der Wissenschaft üblich, er ist Teil des Peer-Review-Verfahrens. Neu ist jedoch, dass Open Science den Kreis der Akteure erweitert, denn wissenschaftliche Diskussionen werden nun auch in offenen Formaten für jedermann zugänglich ausgetragen (z.B. über Tweets bei Twitter).

Aber nicht nur die neuen Kommunikationsformate sind eine Herausforderung. Begünstigt durch die bereits bestehenden gesellschaftlichen und politischen Debatten wurde die wissenschaftliche Arbeit zum Corona-Virus in Deutschland in einigen Medien als interpersonaler Konflikt zweier in Konkurrenz stehender Helden inszeniert - Prof. Drosten und Prof. Streeck (teilweise auch noch in Ergänzung Prof. Kekulé)(siehe beispielhaft Berichte im Merkur und Spiegel). Dieser Erzählung folgend wurde auch in der massenmedialen Berichterstattung zur "Heinsberg-Studie" der Fokus auf den Konflikt gelegt (Vergleiche hierzu auch die Meinung von Dieter Lenzen, Präsident der Uni Hamburg).

Weitere Faktoren mit Blick auf die Akteure machen die Veröffentlichung der "Heinsberg-Studie" in vielerlei Hinsicht zu einem besonderen Ereignis. Verschärft wurden die Umstände dadurch, dass Ministerpräsident Laschet als Auftraggeber der Studie zuvor bereits eine pointierte politische Position bezogen hatte und mutmaßlich auf bestimmte wissenschaftliche Ergebnisse wartete oder zumindest hoffte. Zudem wurden die Ergebnisse in einer Pressekonferenz zusammen mit dem Ministerpräsidenten veröffentlicht, begleitet von einer PR-Kampagne der professionellen Agentur "Storymachine" - ein Vorgehen, das zwar aus der Pandemie-Situation zu verstehen ist, aber für vorläufige Zwischenergebnisse, ohne Einblicke in die Daten oder Veröffentlichung selbst, sehr bemerkenswert ist.

Der Vorwurf "politisch motivierter Auftragsforschung" ist an dieser Stelle durchaus in der Beschreibung zutreffend, aber es ist fraglich, ob sich daraus ein Vorwurf konstruieren lässt. Grundsätzlich gilt festzuhalten, dass Forschung nie frei von Motiven ist, auch vermeintlich unabhängige Grundlagenforschung. Auch sogenannte "Auftragsforschung" an sich stellt kein Problem dar. Freilich muss es dabei um einen Forschungsauftrag, und nicht wie hier unterstellt, um einen "Ergebnisauftrag" gehen. Es gilt allerdings, im besten Open Science-Gedanken, die wissenschaftlichen Prozesse, Methoden und Motivationen offenzulegen und transparent nachprüfbar zu machen. Hier wäre von den Akteuren der "Heinsberg-Studie" mehr Transparenz und Offenheit gefordert und insbesondere eine andere Kommunikationsstrategie nötig gewesen.

In der journalistischen und öffentlichen Bewertung ist mehr Sensibilität für die mediale Inszenierung gefordert, da in der Vergangenheit ein derartiges Bewerben von wissenschaftlichen Erkenntnissen charakteristisch für einige der bekanntesten pseudo-wissenschaftlichen Behauptungen war und die Bezeichnung "Science by Press Conference" geprägt hat: ob 1998 
die falsche Behauptung von Andrew Wakefield, dass Impfungen Autismus auslösen, oder 2012 die fehlerhafte Studie von Gilles-Éric Séralini, die eine krebserzeugende Wirkung gentechnisch veränderter Pflanzen belegen sollte. Sicher erzeugte die Pandemie-Situation eine politische Dringlichkeit, aber gerade bei Fragen der öffentlichen Gesundheit muss ein bedachter Kommunikationsstil gewählt werden

Die heutige Wissenschaft hat dank digitaler Technologien mehr Möglichkeiten, Forschungsprozesse und -ergebnisse zu kommunizieren und Interessierte auch direkt an diesen Prozessen teilhaben zu lassen. Forschende veröffentlichen ihre Arbeiten für alle Open Access, bloggen oder sind auf Social-Media aktiv (wie beispielsweise der extra für die "Heinsberg-Studie" angelegte Twitter-Account). Diese neuen Kanäle und Formate haben nicht nur das Potential, Prozesse schneller und breiter zu kommunizieren, sondern erleichtern auch die Zugänglichkeit zur Wissenschaft. Damit wird ein vormals abgegrenzter, silohafter, innerwissenschaftlicher Diskurs einerseits zugänglich, aber eben auch von verschiedensten nicht wissenschaftlichen Positionen angreifbar. Neue Herausforderungen dabei sind beispielsweise fehlerhafte oder gar absichtlich irreführende, politisch motivierte Auslegungen von wissenschaftlichen Erkenntnissen. Forschung, Politik und Medien sollten sich diesen Herausforderungen stellen. Dazu wird es nicht genügen eine PR Agentur zu engagieren oder wissenschaftliche Ergebnisse im Beisein der politischen Entscheidungsträger - die evidenzbasiert handeln wollen - zu präsentieren. Viel eher müssen die Kompetenzen erweitert werden, wie man Unsicherheiten kommuniziert, wie man das für die Ergebnisse relevante Wissen bereitstellt, sodass es überprüfbar bleibt, und wie man gerade in Krisenzeiten eine wissenschaftliche Debattenkultur pflegen kann ohne sich instrumentalisieren zu lassen.

\title{
Der Umgang mit der "Heinsberg-Studie"
}

\begin{abstract}
Es sind unterschiedliche Ziele und Erwartungen von Wissenschaft und Öffentlichkeit aufeinandergetroffen:

Versäumt wurden dabei zwei Aspekte, die wir in Zukunft besser machen können:
\end{abstract}

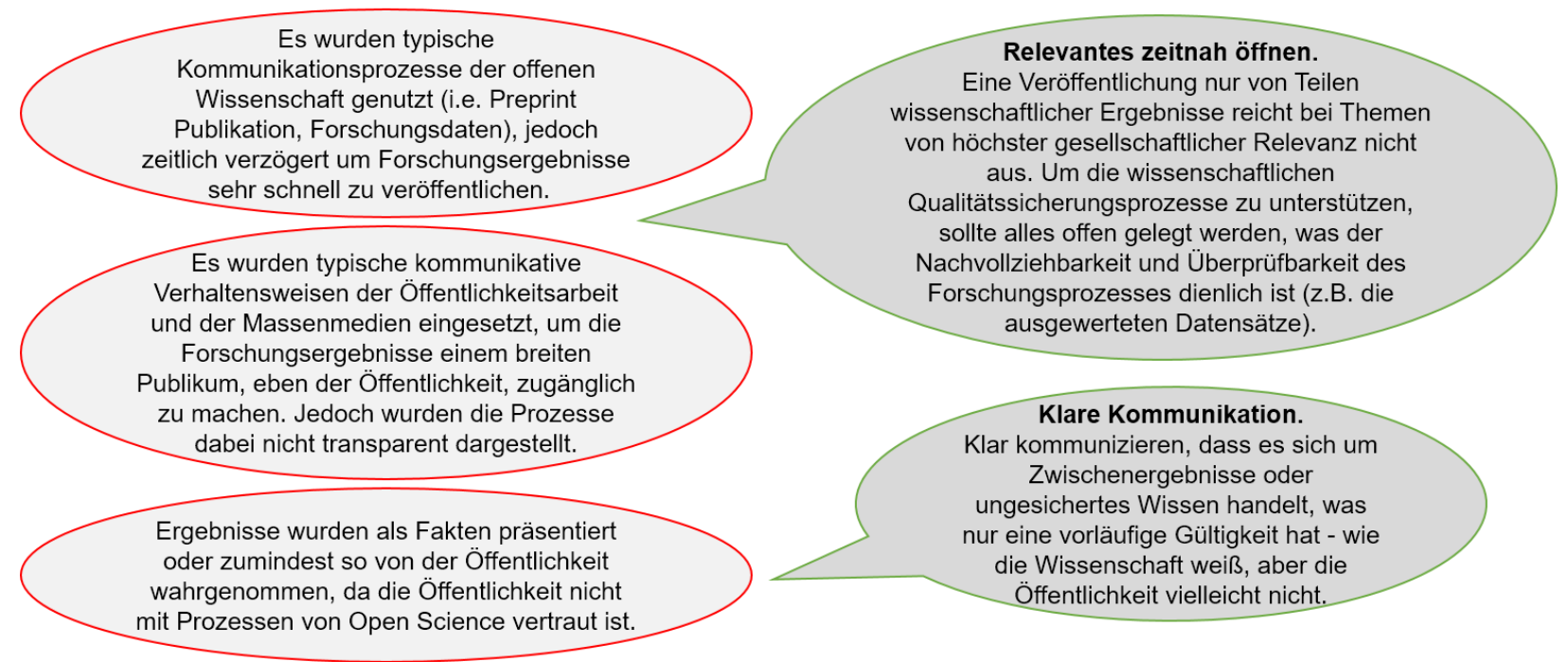

Abb 1. Vorkommnisse und Lehren aus der "Heinsberg-Studie". 


\section{Lessons Learned - Was wir für die Zukunft lernen können}

Wir können aus dem Prozess zur "Heinsberg-Studie" und den entstandenen Diskussionen fünf Lektionen ableiten, die die Wichtigkeit von Open Science und geeigneten Kommunikationsformen in gesellschaftlichen Krisensituationen unterstreichen. Sie sind ein Appell an Wissenschaft und Öffentlichkeit künftig besser im Bereich der Kommunikation zu kooperieren. Das Ziel sollte es sein, die Schnittmengen von Wissen, Erwartungen und Wünschen zwischen Wissenschaft und Öffentlichkeit zu vergrößern und damit ein Hineinversetzen in die jeweils andere Seite zu ermöglichen. Wir sehen Open Science nicht nur als ein hilfreiches, sondern unabdingbares Werkzeug dafür an, diese Schnittstellen zu realisieren.

\section{Lessons Learned 1: Es gibt keine Abkürzung: Wissenschaftliche Kommunikation braucht Sorgfalt und Zeit}

Gerade in Krisensituationen und bei Themen, die die Gesundheit und andere Aspekte höchster gesellschaftlicher Relevanz betreffen, muss besondere Sorgfalt in die Kommunikation von Forschung (z.B. zu wissenschaftlichen Abläufen etc.), (vorläufigen) Forschungsergebnissen und ungesichertem Wissen einfließen. Wissenschaftliche Erkenntnis- und Kommunikationsprozesse lassen sich nicht unendlich beschleunigen, sondern sie brauchen immer eine gewisse Zeit, z.B. in wissenschaftlichen Begutachtungsverfahren (sog. Peer Review), in der ausführlichen Dokumentation des Vorgehens, in der Datenbereinigung oder eben in der Kommunikation von Ergebnissen.

Auch die Einordnung und Darstellungsweise muss sorgfältig gewählt sein. Im Medizinjournalismus haben sich bspw. dafür geeignete Kriterien etabliert. Dazu muss genügend Zeit eingeräumt werden. Zeit, die dem Kommunikationsprozess oftmals nicht zugestanden wird, weil die 'Gier nach Antworten' von Seiten der Politik und der Öffentlichkeit einen viel zu großen Druck aufbaut. Nur durch ausreichend Zeit und besondere Sorgfalt können Wissensbestände sinnvoll offen weiterentwickelt und informierte Entscheidungen getroffen bzw. vertagt werden.

\section{Lessons Learned 2: Ergebnisse vs. Entscheidungen: Es braucht klare Kommunikation von Erwartungen und Leistungen}

Forschende sind es oftmals nicht gewohnt, direkt mit unterschiedlichen Öffentlichkeiten zu kommunizieren. Die Kommunikation innerhalb von Disziplinen folgt wissenschaftskulturellen Mustern, die sich Forschende während Studium und Berufsausübung aneignen. Innerhalb der Disziplinen sind die Erwartungen an die Forschenden und die Kommunikationsprozesse klar. Dies ändert sich aber schon, wenn interdisziplinär kommuniziert wird, d.h. wenn mind. zwei Disziplinen beteiligt sind. In derartigen Forschungsprojekten wird viel Zeit dafür verwendet, die Erwartungen an einander und die jeweiligen Disziplinen zu verhandeln und deutlich zu machen.

Solche Aushandlungs- und Verständigungssprozesse fehlen oftmals in der Kommunikation zwischen Wissenschaft und Öffentlichkeit. Offene Wissenschaft muss mit Nachfragen und Kritik von Seiten der Öffentlichkeit umgehen (lernen). Das erfordert, dass sich beide Kommunikationspartner darauf einlassen und gemeinsam erarbeiten, wie eine derartige Wissenschaftskommunikation aussehen soll. Dazu gehört auch, dass die Wissenschaft erläutert, 
wie Forschungsergebnisse einzuordnen sind, was sie überhaupt leisten kann und an welchen Stellen noch mit unsicherem Wissen umgegangen wird. Dafür darf die Wissenschaft aber nicht unter Beschuss der Öffentlichkeit geraten oder gar politisch instrumentalisiert werden, sondern es sollte stattdessen Raum und Zeit für die Erkenntnis- und Qualitätssicherungsprozesse eingeräumt werden.

\section{Lessons Learned 3: Fehlende Formate: Weiterentwicklung und Nutzung geeigneter Kommunikationsformen}

Die bisherigen Formate der Wissenschaftskommunikation (z.B. journalistische Aufbereitung der Forschungsergebnisse), der innerwissenschaftlichen Kommunikation (z.B. Zeitschriftenartikel) und der Öffentlichkeitsarbeit (z.B. Pressekonferenzen) erfüllen die Anforderungen an sorgfältige und erwartungsgerechte Kommunikation (noch) nicht vollumfänglich. Im Sinne von Open Science bietet es sich hier insbesondere an, digitale Formate (weiter-) zu entwickeln, z.B. Plattformen wie https://outbreaksci.prereview.org/ oder https://prereview.org/, die bislang eher für den innerwissenschaftlichen Austausch genutzt werden und den Qualitätssicherungsprozess öffnen. Solche Plattformen erfüllen aber noch nicht alle Erwartungen, die die Öffentlichkeit an die Wissenschaft hat.

Mittels geeigneter Kommunikationsformate ließe sich auch das Problem adressieren, das mit der Interpretation der Forschungsergebnisse angesichts unsicheren Wissens und komplexer Zusammenhänge einhergeht. Hier, so scheint es, fehlt es in unserer Gesellschaft an Bewusstsein, dass wissenschaftliche Informationen eben nicht immer finale Ergebnisse sein müssen, deren Veröffentlichung aber dennoch richtig und wichtig für die Transparenz und weitere Prüfung ist. Die Zwischenergebnisse sind als nicht das eigentliche Problem, sondern deren Handhabung und Kommunikation. Hier sollte sich auch die Open-Science-Community stärker dafür einsetzen und der Gesellschaft das Bewusstsein von Wissenschaft als dynamischen Prozess näherbringen (Vergleich hierzu den Beitrag von Mafalda Sandrini und Kata Katz).

\section{Lessons Learned 4: Intransparenz schafft Zweifel: Zügige Öffnung von kritischen Bestandteilen des wissenschaftlichen Erkenntnisprozesses}

Man kann nicht oft genug erwähnen, dass vorläufige wissenschaftliche Erkenntnisse umso schneller zu gesichertem Wissen werden, je zügiger sie nachgenutzt und einer kritischen Prüfung unterzogen werden können. Dazu müssen alle kritischen Bestandteile des wissenschaftlichen Erkenntnisprozesses, also alles, was der Beurteilung eines Forschungsergebnisses dienlich ist, transparent verfügbar gemacht werden. Dies ist das Kernziel von Open Science und auch bei der Bekämpfung der Covid19-Pandemie war und ist dies ein wichtiger Aspekt. So erlaubte das Teilen der Gen-Sequenzen des Virus, die zügige Entwicklung von Antikörper-Tests

\section{Lessons Learned 5: Robustes Wissen entsteht gemeinschaftlich}

Eine transparente Nachvollziehbarkeit der am Erkenntnis- und Qualitätssicherungsprozess beteiligten Parteien verdeutlicht zudem, dass Wissenschaft nie ein Ergebnis von Einzelaktivitäten, sondern immer eine kollektive und im besten Fall kollaborative Unternehmung ist. Werden 'bahnbrechende' wissenschaftliche Ergebnisse nur von einem Forschungsteam 
präsentiert oder losgelöst von der wissenschaftlichen Diskussion, dann sollte man dies mit Vorsicht betrachten. Denn: Wissenschaftliche Ergebnisse stehen in einem komplexen Zusammenhang mit vorhergehender und zukünttiger Wissensproduktion. Was in den Medien mitunter als "Streit" unter Forschenden deklariert wird, ist ein notwendiger Prozess der Erkenntnissicherung und beruht auf dem Prinzip des Peer-Review. Wissenschaftliche Ergebnisse können nur innerhalb dieses Zusammenhangs interpretiert und durch Kollaboration zu gesichertem Wissen werden.

Wissenschaft und wissenschaftlicher Fortschritt sind grundlegend auf das Teilen von Wissen, also Open Science, angewiesen. In Krisensituationen sind traditionelle Formate wie Artikel in Fachmagazinen aber zu langsam, daher müssen andere Formate gefunden werden (siehe Lesson 3), die u. U. auch schon einen Einblick in den Forschungsprozess erlauben. Gerade in Krisenzeiten sollte gemeinschaftlich gesichertes Wissen als Allgemeingut behandelt werden. Uns ist klar, dass Lesson 1 und Lesson 4 in einem gewissen Spannungsverhältnis zueinander stehen, doch wir sind sicher, dass eine konsequente Umsetzung von gut durchdachten Open Science Praktiken diese Spannungen produktiv werden lässt. 